BU-TH/96-2 ; IISc-CTS-7/96 ; TIFR/TH/96-21

hep-ph/9605460

\title{
CAN THE ALEPH FOUR-JET EXCESS BE EXPLAINED IN A SUPERSYMMETRIC MODEL WITH R-PARITY VIOLATION?
}

\author{
Dilip Kumar Ghosh ${ }^{a}$ \\ Department of Physics, University of Bombay, \\ Vidyanagari, Santa Cruz (East), Mumbai 400 098, India. \\ Rohini M. Godbole 円 $^{b}$ \\ Centre for Theoretical Studies, Indian Institute of Science, \\ Bangalore 560 012, India. \\ Sreerup Raychaudhuri ${ }^{c}$ \\ Theoretical Physics Group, Tata Institute of Fundamental Research, \\ Homi Bhabha Road, Mumbai 400 005, India.
}

\begin{abstract}
We investigate the possibility that the excess of four-jet events in $e^{+} e^{-}$collisions at LEP-1.5 which has been reported by the ALEPH Collaboration could be due to the production of charginos or neutralinos, followed by their decay into quark jets through baryon numberviolating $\left(\lambda^{\prime \prime}\right)$ couplings. An estimate at the parton level shows, however, that these events cannot be due to neutralinos because of the low cross-section, and is unlikely to be due to the production of chargino pairs because of the largely different event shapes.
\end{abstract}

Pacs. Nos: 14.80.Ly, 13.65.+i, 12.60.Jv

Electronic address: ${ }^{a}$ dghosh@theory.tifr.res.in; $\quad{ }^{b}$ rohini@cts.iisc.ernet.in; $\quad{ }^{c}$ sreerup@theory.tifr.res.in

\footnotetext{
${ }^{1}$ on leave of absence from the Department of Physics, University of Bombay, Mumbai, India.
} 


\section{INTRODUCTION.}

In the last few months, considerable interest has been generated by fresh results coming from the LEP Collider at CERN. Upgradation of the collision energy to 130/136 GeV has given rise to expectations that one might see new physics effects at this energy. However, most of the results obtained till now at this LEP-1.5 collider are consistent [1] with the predictions of the Standard Model (SM) and merely lead to new constraints on physics beyond it. There is, however, one notable exception to this trend. The ALEPH Collaboration has reported [2] an excess over the SM prediction in the $e^{+} e^{-} \rightarrow$ four-jets channel. The number of events in the data sample of $5.7 \mathrm{pb}^{-1}$ which satisfy the rather stringent criteria imposed for this search is 16 , which is significantly in excess of the 8.6 events predicted by the SM. Of these 16 events, 9 have a four-jet invariant mass of about $105 \mathrm{GeV}$. The rest are consistent with the SM background.

This observation is rather hard to interpret. Unless the observed result is due to a statistical fluctuation, which is somewhat remote in view of the low estimated probability of $10^{-4}$, it seems natural to assume that both the measurement and the SM prediction are correct and hence, the four-jet excess is a genuine new physics effect. While this may seem to be unduly optimistic at this preliminary stage of the upgraded LEP runs, it is nevertheless amusing to probe new physics scenarios which could lead to a four-jet excess. One such scenario is discussed in this article, namely, supersymmetry with violation of $R$-parity as a possible candidate for this effect.

The basic idea expanded in our work is rather simple. We consider two possibilities within the framework of the Minimal Supersymmetric Standard Model (MSSM) with $R$ parity violation [3, 团.

1. The lightest neutralino, which is assumed to be the lightest 4 supersymmetric particle (LSP), may have mass around $50-55 \mathrm{GeV}$ and be pair-produced in $e^{+} e^{-}$collisions at LEP-1.5. If $R$-parity is not conserved, these neutralinos could decay [5] and the invariant mass of the decay products of each would peak around $50-55 \mathrm{GeV}$. The

\footnotetext{
${ }^{2}$ This is natural in a $R$-parity conserving scenario, but is not essential if $R$-parity is violated; however, we keep this configuration since it involves the minimum change from the familiar MSSM scenarios.
} 
sum of these invariant masses would then peak around $105 \mathrm{GeV}$. If $R$-parity is violated through baryon number-violating couplings $\left(\lambda^{\prime \prime}\right)$, the neutralinos will decay into three quark jets each, which could then merge to give the distinctive four-jet signals.

2. The lighter chargino (which is assumed to be heavier than the lightest neutralino) may have mass around $50-55 \mathrm{GeV}$ and be pair-produced similarly at LEP-1.5. As before, when the charginos decay, the sum of invariant masses of the decay products of both would be expected to peak in or around $105 \mathrm{GeV}$. We then envisage the decay of each chargino to a neutralino (LSP) and a pair of quark jets; this LSP then decays through $\lambda^{\prime \prime}$ couplings into three jets. Thus one has ten jets in all, which could then merge to give four-jet signals. We could also, in principle, expect signals in channels with more jets, though, as it turns out, these are not really significant.

Recently, it has been pointed out [6] that it is possible for the chargino to decay directly into jets through $R$-parity violating $\lambda^{\prime \prime}$ couplings without the intermediacy of a neutralino as considered in 2. This is an exciting possibility but we do not pursue it here; our assumption being that the neutralino is suficiently lighter than the chargino and that the relevant $\lambda^{\prime \prime}$ coupling is too small for the direct chargino decay to compete with the usual MSSM decay mode. This is consistent with our philosophy of keeping the changes from the conventional MSSM scenario to a minimum.

We analyse the above signals using a parton-level Monte Carlo event generator to scan the MSSM parameter space. The use of a parton-level generator has two advantages: $(a)$ it is quick, so that a detailed study of the parameter space is possible; and (b) the analysis is relatively simple so that one can focus on the basic physics issues. On the other hand, this approach has the obvious drawback that the algorithms used to analyse jets are necessarily crude. Hence our results should be considered diagnostic only. The issues which have been addressed in this work are confined to checking if the processes under consideration are at all viable and (if such is the case) to identify the relevant part of the MSSM parameter space. In case of a viable signal, our investigation could, in principle, aid further studies of the process in which the analysis of jets is done in greater detail.

Before we pass on to the details of our analysis, it would be appropriate to discuss in 
greater detail the nature of the multijet events we are analysing. The events seen by the ALEPH Collaboration [2] consist of spherically distributed multijet events where the event shapes are consistent with a purely hadronic final state. All events satisfy the requirement that the net visible energy of the jets is at least $70 \%$ of the centre-of-mass energy at LEP-1.5. Moreover there are practically no displaced vertices in the microvertex detector, indicating that the jets originate from light quarks or gluons rather than $b$-quarks.

To select these events out of the SM multijet background, the ALEPH Collaboration has imposed a number of kinematic and other cuts on the jets. The principal background comes from the process $e^{+} e^{-} \rightarrow q \bar{q}$ followed by gluon radiation from the quarks. Most of the gluon jets, however, will be soft and much of this background is eliminated when the following selection criteria are imposed:

1. At least eight charged particle tracks must be reconstructed with at least four hits in the time projection chamber, with a polar angle $\theta$ with respect to the beam such that $|\cos \theta|<0.95$ and originating within a cylinder of length $20 \mathrm{~cm}$ and radius $2 \mathrm{~cm}$ coaxial with the beam and centred at the nominal collision point.

2. The scalar sum of the charged particle momenta must exceed $10 \%$ of the centre-of-mass energy.

3. Radiative $Z^{0}$ returns are removed by requiring the missing momentum measured along the beam direction to be smaller than $0.75\left(m_{\text {vis }}-90 \mathrm{GeV}\right)$, where $m_{\text {vis }}$ is the invariant mass of the system formed by all energy-flow particles.

4. Events with fewer than four jets are rejected.

5. None of the jets (in the four jets sample) contains more than $80 \%$ electromagnetic energy.

6. The dijet invariant mass of each pair of jets in the four-jet sample (6 pairs in all) is not less than $25 \mathrm{GeV}$;

7. The sum of pairs of jet invariant masses is not less than $10 \mathrm{GeV}$;

8. Each pair of jets has a minimum of 10 charged tracks between them. 
It should be noted that the ALEPH Collaboration observe no six-jet events and all fivejet events have been converted into four-jet events by merging the pair of jets which has the minimum dijet invariant mass. They report the observation of 16 events which satisfy all these criteria against a SM prediction of 8.6 events. What is even more interesting, however, is the distribution in four-jet invariant mass. If the jets are numbered 1,2,3,4 and $\Delta M \equiv \min \left|M_{i j}-M_{k l}\right|$ out of the combinations $i j ; k l=12 ; 34$ or $13 ; 24$ or $14 ; 23$ respectively, then for 9 of these events the four-jet invariant mass, defined as the sum $\Sigma M \equiv\left(M_{i j}+M_{k l}\right)$ for the combination $i j ; k l$ which yields $\Delta M$, lies within $102.1-108.4 \mathrm{GeV}$. The predicted $\mathrm{SM}$ background in this bin is about 0.8 events for $5.7 \mathrm{pb}^{-1}$ luminosity. The probability of this accumulation being due to a fluctuation in the SM background is as estimated to be as low as $1.1 \times 10^{-4}[2]$.

In section 2 we discuss the decay of the LSP in the $R$-parity violating model of our choice and explain our techniques for analysing multijet signals. Section 3 analyses the possibility that the four-jet anomaly may be due to neutralinos. In section 4 we make a similar discussion for charginos. Finally, section 6 contains a summary of our results and conclusions.

\section{LSP DECAY WITH BARYON NUMBER VIOLATION.}

The crucial feature of our analysis is the decay of the neutralino (assumed to be the LSP) into jets through $R$-parity violating couplings. These couplings can, in general, be

of two kinds: those that violate lepton number and those that violate baryon number. If both are present, the theory predicts a large width for proton decay which is inconsistent with current data [7]. Accordingly, one has to assume that either lepton or baryon numberviolating couplings are present, but not both. For the purposes of this work, we assume that there are no lepton number-violating couplings and concentrate on baryon number-violation only. The baryon number-violating term in the superpotential has the form [3]

$$
\mathcal{W}_{B B}=\sum_{i j k} \lambda_{i j k}^{\prime \prime} \hat{U}_{i}^{c} \hat{D}_{j}^{c} \hat{D}_{k}^{c}
$$

where $\hat{U}^{c}, \hat{D}^{c}$ are chiral superfields containing the right-handed $u, d$-quarks and the indices 
$i, j, k$ run over the three quark generations. This leads to the interaction Lagrangian

$$
\mathcal{L}_{B B}^{i n t}=-\sum_{i j k} \lambda_{i j k}^{\prime \prime}\left[\widetilde{u}_{R i}^{*} \bar{d}_{R k} d_{L j}^{c}+\widetilde{d}_{R j}^{*} \bar{u}_{R i} d_{L k}^{c}+\widetilde{d}_{R k}^{*} \bar{d}_{R j} u_{L i}^{c}\right]+H . c .
$$

Though colour indices are not explicitly shown, the interaction term must be a colour singlet; this requires the $\lambda_{i j k}^{\prime \prime}$ to be antisymmetric in the last two indices.

One can now envisage the decay of a LSP into a quark and an off-shell squark which then goes to a pair of quarks through the above baryon number-violating couplings [5]. There are three possible diagrams corresponding to the three terms in the interaction Lagrangian and these are shown in Figure 1. For this study, we assume that the coupling $\lambda_{212}^{\prime \prime}$ is dominant and that the others may be neglected. This is not an essential requirement of the theory, but is the simplest option [8]. However, it is important to note that the decay width is proportional to the square of this coupling $\lambda_{212}^{\prime \prime}$ only, so that the coupling cancels out of numerator and denominator in the branching ratio. In any case, since this is the only decay mode of the LSP in the scenario under consideration, the branching ratio of the LSP to jets is unity. It is also important to note that the $\lambda_{212}^{\prime \prime}$ coupling ensures that there are no $b$-jets in the final sample, which is what the ALEPH Collaboration finds?.

Till the present date, no direct search has been made at LEP-1 for $R$-parity violating signals in the presence of $\lambda^{\prime \prime}$ couplings, though corresponding searches have been made assuming the presence of $\lambda$ couplings [9] and strategies have been discussed for $\lambda^{\prime}$ and $\lambda^{\prime \prime}$ couplings [10]. Thus, there are no direct bounds from LEP-1 data on MSSM parameters in this scenario. Such a study is, however, possible, and is in progress [11. Moreover, since the LSP decays (into jets), the usual missing energy signals are not expected to be seen. In view of this, the only constraint coming from LEP-1 data is the requirement that the contribution of LSP pair-production to the total $Z$-width should be consistent with the SM prediction and the experimental error [12]. There is also a strong bound from the nonobservation of charginos at LEP-1 leading to the requirement that the mass of the chargino should exceed $45 \mathrm{GeV}$; however, this constraint affect roughly the same region of parameter space as the previous one, since the chargino contribution to the $Z$-total width is large. Furthermore, for $M_{\tilde{g}} \lesssim 150 \mathrm{GeV}$, one should have [13] seen spectacular multijet signals at LEP-1, which is not

\footnotetext{
${ }^{3}$ Actually one of the 16 events does contain [2] $b$-jets, but this could well arise from the QCD background.
} 
the case. However, one certainly needs to consider a somewhat larger allowed region in the MSSM parameter space than is the case for $R$-parity conserving models.

Since the decay of the LSP will lead to final states with several quark jets, we now explain our method of determining the number of distinct jets. As explained before, we use a simple parton-level Monte Carlo event generator which is unable to simulate the details of the hadronic fragmentation. We therefore make the somewhat crude approximation that the direction of the parent parton is the same as that of the thrust axis of the resulting jet and that the hadronic material is confined to a cone around it. Thus, if the directions of two partons which engender jets are separated by $\sqrt{\Delta \eta^{2}+\Delta \phi^{2}} \equiv \Delta R \leq 0.7$, we consider the jets to have merged. While we recognise that this approximation is a somewhat crude one, it is a commonly used rule of thumb in analyses of jets produced around the electroweak scale, at least at hadron colliders 14. We have also considered the Durham algorithm (used by the ALEPH Collaboration) for the jet-merging procedure, merging partons with $y<0.008$ rather than the fixed cone algorithm discussed above, but the final results do not change by more than a few percent from the results presented in this work (using the cone algorithm). Once a pair of jets is considered to have merged, the momentum and thrust axis of the resultant jet are constructed simply by vectorially adding the three-momenta of the original jets.

In our analysis we consider the production of a LSP pair or a chargino pair and their subsequent decay to multijet final states. These are allowed to pass through the 'jet'-merging algorithm described above and the reconstructed 'jets' (by which we mean one or more partons, or more specifically, quarks) are passed successively through the following kinematic cuts, which more-or-less follow those used by the ALEPH Collaboration in their analysis:

1. The jets should have rapidity less than 3 , since this roughly corresponds to the polar angle cut used by the ALEPH Collaboration. It turns out, however, that most of the jets which contribute to the final signal have rapidity $\leq 2$, so we could easily accommodate a more stringent rapidity cut without affecting the signal. This may ultimately be required in view of the lower detection efficiency for high rapidities.

2. The scalar sum of momenta of the jets in the final analysis must be greater than 0.1 
of the $e^{+} e^{-}$centre-of-mass energy, i.e. $13 \mathrm{GeV}$;

3. The sample should contain four or more jets only. All five-jet events are converted into four-jet events by merging the pair of jets with the minimum invariant mass. Events with six or more are jets are counted separately.

4. The dijet invariant mass of each pair of jets in the four-jet sample is greater than 25 $\mathrm{GeV}$.

In the absence of detector simulation, calorimetic cuts cannot be imposed. Moreover, in our parton-level event generator, we are unable to impose constraints which involve the invariant masses of individual jets or the multiplicity of charged tracks, since that would be possible only in a simulation which takes into account the hadronisation processes. Accordingly, we make the ad hoc assumption, in the following discussion, that the supersymmetric signal is reduced by $40 \%$ by cuts of this nature. The choice of this factor is guided by the reduction in the signal and background presented by the ALEPH Collaboration, which are $27 \%$ and $47 \%$ respectively. While we recognise that this is a rather crude approximation, it is not likely to affect the conclusions of our study, as we shall see. It should be noted that we have not included this reduction factor in the kinematic distributions shown in this work; these would accordingly be reduced and probably smeared further by application of these cuts in a simulation which takes account of fragmentation.

\section{FOUR-JET EXCESS FROM NEUTRALINO PAIR-PRODUCTION.}

We first consider the scenario when the four-jet signal is due to the production of a pair of neutralinos (LSP's). For this, one requires the mass of the LSP to lie in the range allowed by LEP-1 data and accessible to LEP-1.5. This automatically restricts our analysis to a limited region in the MSSM parameter space obtained by variation of gluino mass $\left(M_{\tilde{g}}\right)$, Higgsino mixing parameter $(\mu)$ and the ratio of vacuum expectation values of the two Higgs doublets $(\tan \beta)$. Dependence on the squark mass is minimised by considering the gluino mass evaluated at the electroweak scale, i.e. $M_{\tilde{g}}\left(M_{Z}\right)$. Of course, in this work, we have made the assumption of gaugino mass unification at a high scale, which enables us to use 
the gluino mass as a parameter of the electroweak gaugino sector; however, the analysis would be unchanged if we relaxed this hypothesis and used instead the soft-SUSY breaking parameter $M_{2}$ in the $S U(2)$ sector.

The cross-section for production of a pair of neutralinos has been calculated in the MSSM, in terms of the above parameters, by a number of authors 15. We have checked that our cross-sections are consistent with theirs, both analytically and numerically. It may be noted that neutralinos are produced through $s$-channel $Z$ exchanges as well as $t, u$-channel $\widetilde{e}_{L}, \widetilde{e}_{R}$ exchanges and hence are rather sensitive to the masses of the selectrons for the case when the neutralino is gaugino-dominated. In fact, the cross-sections fall as the selectron masses go up. As we shall see presently, the predicted number of four-jet events from neutralino pair-production is small, so it is desirable, for our purposes, to choose the parameters to maximise the cross section. This is achieved by choosing the selectron masses as light as possible. We have chosen the left-selectron mass consistent with a sneutrino mass of $60 \mathrm{GeV}$; the right selectron is also chosen to have a mass of $60 \mathrm{GeV}$. These values are more or less at the edge of the allowed range [1].

The decays of the LSP are mediated by right-squark exchanges and hence one has a nominal dependence on the relevant squark masses too. This is rather weak, however, because, the branching ratio being unity, the only effect of increasing the squark mass is to change the kinematic distributions of the decay products. After merging of jets, however, much of this effect — such as it is - is washed out. Consequently, the final cross-section has very little dependence on the squark mass, especially when the value becomes significantly larger than the neutralino mass. This is always the case if the squark mass is taken as $150 \mathrm{GeV}$ or above. In our analysis we set the squark mass to $300 \mathrm{GeV}$ for definiteness.

At this point, it might be worth mentioning that a much lower value of squark mass is probably consistent with $\mathrm{CDF} / \mathrm{D} 0$ bounds in a scenario in which $R$-parity is violated. Current CDF/D0 bounds [18] on squark as well as gluino masses are derived from signals which trigger on missing energy and momentum and may be considerably relaxed [16] if the LSP decays, especially in the case where baryon-number is violated. Limits on squark and gluino masses from $\mathrm{CDF} / \mathrm{D} 0$ data in the case of $R$-parity violation with $\lambda^{\prime \prime}$ couplings have not, in fact, been investigated thoroughly, though such an analysis is, in principle, possible 
[17. In any case, for the present analysis, squark masses are set rather high, so we have not exploited the absence of a bound. On the other hand, we have used gluino masses in the ranges allowed by LEP-1 without reference to CDF/D0. Of course, one could relax the assumption of gaugino mass unification at a high scale, in which case the gluino mass would become irrelevant as a parameter.

The results of our analysis of the four-jet signal arising from neutralinos of the appropriate mass is shown in Figure 2 as a scatter plot of the predicted number of four-jet events versus the mass of the neutralino (LSP). Each point in the scatter plot corresponds to a different set of $\left(M_{\widetilde{g}}, \mu, \tan \beta\right)$ in the ranges $M_{\widetilde{g}}=0.1$ to $1 \mathrm{TeV}$ (in steps of $10 \mathrm{GeV}$ ), $\mu=-1$ $\mathrm{TeV}$ to $1 \mathrm{TeV}$ (in steps of $25 \mathrm{GeV}$ ) for $\tan \beta=1.5,2,5,10,15,20,25,30,35$ respectively, subject to constraints arising from LEP-1 data and kinematic accessibility to LEP-1.5. For this plot, we have taken the sneutrino mass to be $60 \mathrm{GeV}$ for reasons explained above and set the masses of squarks belonging to the first two generations to $m_{\widetilde{q}_{L}}=m_{\widetilde{q}_{R}}=300 \mathrm{GeV}$. It may be noted that the number (which assumes $5.7 \mathrm{pb}^{-1}$ integrated luminosity at $\sqrt{s}=130$ $\mathrm{GeV}$ ) of four-jet events never rises above 1.8 events which, added to the SM background of 8.6 events, barely touches 10.4 events and never approaches anywhere near the ALEPH observation of 16 events. It is quite clear, therefore, that the pair-production of neutralinos followed by their $R$-parity violating decays cannot be the explanation of the observed excess in four-jet events. This is a fairly robust result despite the crudity of our parton-level analysis, since it is hard to see a more refined analysis changing the result by a factor of 4 or more, which would be required to explain the observed events.

\begin{tabular}{|c|c|c|c|}
\hline$\left(m_{\tilde{g}}, \mu, \tan \beta\right)$ & $(340,-400,2)$ & $(360,-300,15)$ & $(370,300,30)$ \\
\hline$M_{\widetilde{\chi}_{1}^{0}}$ & $51.9 \mathrm{GeV}$ & $51.9 \mathrm{GeV}$ & $51.8 \mathrm{GeV}$ \\
\hline produced neutralino pairs & 6.13 & 5.75 & 5.59 \\
\hline$\Sigma \mathbf{p}_{\text {jet }} \geq 0.1 \sqrt{s}$ & 6.13 & 5.75 & 5.59 \\
\hline$\geq 4$ jet events only & 3.32 & 3.10 & 3.01 \\
\hline$M_{i j} \geq 25 \mathrm{GeV}$ & 2.09 & 1.96 & 1.90 \\
\hline Multiplicity \& jet masses & $\sim 1.25$ & $\sim 1.18$ & $\sim 1.14$ \\
\hline
\end{tabular}

Table 1: Illustrating the effect of various cuts on the neutralino induced multijet signal of 
Figure 2 for specimen points in the parameter space.

The numbers displayed in Figure 2 may seem unexpectedly small, in view of the fact that the neutralino production cross-section can be as large as $1-1.5 \mathrm{pb}$ at LEP-1.5 [15]. The explanation for this lies in the selection criteria imposed by the ALEPH Collaboration. The effects of these criteria are illustrated in Table 1 for three specimen points in the parameter space where the cross-section is relatively large and the neutralino mass lies around $52 \mathrm{GeV}$, which means the distribution in the sum of dijet invariant mass would be peaked around 104 GeV. Initially, about 6 neutralino-induced events are indeed predicted. The cut removing soft jets makes no impact on the signal. This is easy to justify using simple kinematical arguments. However, the channels with four or more jets retain only about half of this cross-section. This is, in turn, further reduced by one-third by the requirement that the dijet invariant mass of each pair of observed jets be greater than $25 \mathrm{GeV}$. The signal is already down to about 2 events and can be expected to fall to barely more than 1 event by application of cuts on charged track multiplicity and jet invariant mass.

Finally, it may be noted that the effects of initial state radiation can, in general, increase the neutralino pair-production cross-section [2] because the effective centre-of-mass energy then falls back near the $Z$-resonance; however this drives us closer to the threshold for production of neutralinos so that the cross-section undergoes some phase-space suppression. The net result of these opposing effects is, in general, to keep the cross-section just so. In any case, however, the final numbers for neutralino production are so small that this point is merely academic.

\section{FOUR-JET EXCESS FROM CHARGINO PAIR-PRODUCTION.}

We now turn to the other possibility that the four-jet signals arise from the production of a pair of (lighter) charginos of mass in the range $46-65 \mathrm{GeV}$ which is allowed by LEP-1 data and accessible to LEP-1.5. Each chargino decays to a neutralino (LSP) and an off-shell W-boson which then goes to a pair of jets or a pair of leptons. The neutralino then decays (as before) to three jets. Either of the following things can happen: 
1. Both off-shell $W^{*}$ 's can decay to hadrons, making five jets in all from each chargino. The ten jets in the final state can then merge to give four-jet events.

2. One off-shell $W^{*}$ can decay to leptons and the other to hadrons. The leptons can evade detection if they lie within the jets coming from the decay of the neutralino and the other $W^{*}$. After merging of jets, this configuration, too, can yield some four-jet events.

3. One off-shell $W^{*}$ can decay to leptons and the charged lepton can be isolated from the jets, leading to a signal with a hard isolated lepton (electron or muon), missing energy and multijets.

4. Both off-shell $W^{*}$ s can decay to leptons leading to a signal with a pair of hard leptons (electrons or muons) of opposite sign, large missing energy and multijets.

Of these, the first two will contribute to the four-jet excess observed by ALEPH. They can also, in principle, lead to multijet events with higher multiplicity than the four- and five-jet events studied by ALEPH, since, after all, we start with ten jets. The other two options will lead to clear signals which should be observable not only by ALEPH but also by the other detectors at LEP, provided the signal is large enough. This would constitute an extra test of the scenario under consideration, provided it proves workable in the first place.

Like the cross-sections for neutralino production, the cross-sections for chargino pairproduction are also well known and we have checked that our results are in agreement, both analytically and numerically, with those of earlier authors [19]. As the predicted crosssections for chargino production are much larger than those for neutralinos, we get a sizable residue after application of all the relevant cuts. The decays of the chargino to a neutralino and a pair of light quarks are $R$-parity conserving and again have been studied before [20]. The novel feature of our analysis is simply the decay of the neutralino (LSP) into jets.

In order to have charginos of mass in the appropriate range, we are, as before, restricted to a part of the MSSM parameter space. This corresponds to the region between the solid and dotted lines (for each $\tan \beta$ ) in Figure 3. We have not included CDF/D0 bounds in this figure for reasons explained above. Thus, we consider a fairly large part of the parameter space which supports the relevant masses of the lighter chargino. 
The chargino production cross-section has contributions from $s$-channel $\gamma, Z$ exchanges and $t$-channel $\widetilde{\nu}$ exchange. The variation of the chargino cross-section with the mass of the sneutrino is given, for a fixed set of other MSSM parameters, in Figure 4 . The $s$ and $t$ channel contributions are known [19] to interfere destructively, leading to a dip in the crosssection for some values of the sneutrino mass when the charginos are gaugino-dominated, as is the case in this figure. The dip arises in the region $m_{\widetilde{\nu}} \simeq 30-40 \mathrm{GeV}$, which is ruled out by LEP-1 data. Thus, in the allowed region, the cross-section essentially grows with sneutrino mass, with a tendency to saturate as the mass goes as high as a few hundred GeV. In this work, we shall require a somewhat small chargino production cross-section, so that it becomes desirable to choose a sneutrino mass at the lower end. We choose $65 \mathrm{GeV}$, which is marked by a bullet in the figure and corresponds to a cross-section of about $8 \mathrm{pb}$.

We then consider decays of the charginos to LSP's and jets and/or non-isolated leptons, followed by decays of the LSP's to jets. This involves, as before, a nominal dependence on the squark mass, which, is, however, weak, as in the case of neutralino production. We set the squark mass to $500 \mathrm{GeV}$ in the subsequent analysis. The ten (or eight) jets in the final state are passed (as in the neutralino case) through our simple-minded jet-merging algorithm to determine the number of distinct jets. These results are illustrated in Figure 5 which is a scatter plot of the predicted number of four-jet events for chargino masses in the range 45 $-65 \mathrm{GeV}$ for different values of the MSSM parameters. As in Figure 2, each point in the scatter plot corresponds to a different set of $\left(M_{\widetilde{g}}, \mu, \tan \beta\right)$ in the ranges $M_{\tilde{g}}=0.1$ to $1 \mathrm{TeV}$ (in steps of $10 \mathrm{GeV}$ ), $\mu=-1 \mathrm{TeV}$ to $1 \mathrm{TeV}$ (in steps of $25 \mathrm{GeV}$ ) for $\tan \beta=1.5,2,5,10$, 15, 20, 25, 30, 35 subject, as before, to constraints imposed by LEP-1 data and accessibility at LEP-1.5. Again, as beforem no CDF/D0 constraints are imposed. The points marked by bullets in Figure 3 are selected out of Figure 5 by imposing the conditions that the number of four-jet events is $(16 \pm 0.5)$ and $50 \mathrm{GeV}<M_{\tilde{\chi}_{1}^{+}}<55 \mathrm{GeV}$. It should be noted that the bullets correspond to some value of $\tan \beta$ among the listed values; not necessarily one of the values marked on the contours. Thus, points which lie in the region ruled out for $\tan \beta>5$ correspond to $\tan \beta=1.5$ or 2 .

It may be seen that the events are fairly densely clustered in an arc which gradually goes down as the chargino mass increases. This is indicative of phase-space suppression 
rather than a diminishing coupling. For this figure, the sneutrino mass has been tuned to $65 \mathrm{GeV}$ in order to ensure that the number of four-jet events consistent with the ALEPH observation should be compatible with a chargino mass of $50-55 \mathrm{GeV}$, at least for the range where the points are most thickly clustered. Since there are many points above this region, it should be possible to decrease the sneutrino mass (this decreases the cross-section as shown in Figure 4) further. However, if one increases the sneutrino mass, the cross-section and hence the number of four-jet events goes up and we are then confined to just a few points in the parameter space which would give the required mass of the chargino and the required number of events. It may be concluded, then, that the chargino solution to the four-jet excess problem favours a light sneutrino with $m_{\widetilde{\nu}} \sim 60-70 \mathrm{GeV}$ (though it does not demand this absolutely). What is important, however, is that one can find at least a tentative explanation for the ALEPH excess in terms of chargino pair-production.

Table 2

\begin{tabular}{|c|c|c|c|}
\hline$\left(m_{\tilde{g}}, \mu, \tan \beta\right)$ & $(160,-600,2)$ & $(200,500,15)$ & $(200,400,30)$ \\
\hline$M_{\widetilde{\chi}_{1}^{ \pm}}$ & $53.7 \mathrm{GeV}$ & $54.5 \mathrm{GeV}$ & $54.3 \mathrm{GeV}$ \\
\hline$M_{\widetilde{\chi}_{1}^{0}}$ & $25.0 \mathrm{GeV}$ & $28.2 \mathrm{GeV}$ & $28.3 \mathrm{GeV}$ \\
\hline produced chargino pairs & $30.0(9.4)$ & $27.9(9.5)$ & $28.3(9.7)$ \\
\hline$\Sigma \mathbf{p}_{\text {jet }} \geq 0.1 \sqrt{s}$ & $30.0(9.4)$ & $27.9(9.5)$ & $28.3(9.7)$ \\
\hline$\geq 4$ jet events only & $18.5(4.8)$ & $17.5(5.2)$ & $17.8(5.4)$ \\
\hline$M_{i j} \geq 25 \mathrm{GeV}$ & $12.8(2.8)$ & $12.7(3.5)$ & $12.8(3.5)$ \\
\hline Multiplicity \& jet masses & $\sim 7.7(1.7)$ & $\sim 7.6(2.1)$ & $\sim 7.7(2.1)$ \\
\hline+ SM background & $\sim 16.3$ & $\sim 16.2$ & $\sim 16.3$ \\
\hline
\end{tabular}

Table 2: Illustrating the effect of various cuts on the chargino-induced multijet signal of Figure 5 for candidate points in the parameter space. Numbers in parantheses show the contribution from events where the final state contains a lepton which goes undetected.

In Table 2, we show the effect of different kinematic cuts for three points in the parameter space where the cross-section is consistent with the ALEPH observation and with a chargino mass of around $54 \mathrm{GeV}$. These effects are rather similar to those in Table 1 . We start with 
about 30 events. Once again, the cut removing soft jets makes no impact on the signal (the reasons are the same as before) and the four-or-more-jet channels contain about 60 $\%$ of the signal. The requirement that each dijet invariant mass be greater than $25 \mathrm{GeV}$ reduces this to about $43 \%$ and the final reduction by about $40 \%$ due to multiplicity and jet invariant mass cuts brings the signal down to the required level, which is about $25 \%$ of the original cross-section. Events where there is a lepton which goes undetected because of its non-isolation from the nearest jet make up about a quarter of the excess contribution.

It is interesting that the jet merging algorithm makes the four-jet channel the dominant one, though some three-jet and five-jet events are also predicted. This is illustrated in Figure 6. Just a single six-jet event is predicted - which is consistent with the ALEPH observation of none, in view of the low statistics. The five-jet events are subsequently converted to four-jet events in our analysis, following the ALEPH Collaboration. The three-jet events, of course, have large QCD backgrounds. The fact that the ten jets from chargino decay merge to give multijet signals which peak for precisely four-jets is a somewhat unexpected result and is one of our most important observations. It is worth mentioning at this juncture that the use of the Durham algorithm for jet-merging does not change this conclusion.

It is also noteworthy that the region in parameter space which gives a viable cross-section for chargino pair-production leads to a LSP mass of $25-28 \mathrm{GeV}$. As explained before, this is constrained, by the total $Z$-width only because of the presence of $\lambda^{\prime \prime}$ couplings. Hence such a low value for the LSP mass will be allowed in our scenario under currently available LEP-1 and LEP-1.5 constraints. However, it is perhaps worth mentioning that with this LSP mass one would predict 2.1, 7.6, 7.9, 3.6, 0.5 events in the 1, 2, 3, 4, 5-jet channels respectively for parameters corresponding to the first column of Table 2 (the numbers are quite similar for the other two columns). These jets would not affect the signal in Table 2 because of the lower invariant mass of the jets, but might possibly be observable (though the QCD backgrounds would also be significant). It would be interesting to conduct such a search within a more general study of supersymmetry signals in the presence of $\lambda^{\prime \prime}$ couplings. Such a study has, in fact, been taken up [11].

Let us now address the important question of event shapes in the scenario discussed in this work. The parameters are chosen such that the mass of the produced chargino is around 
$52-54 \mathrm{GeV}$. One should, therefore, expect the four-jet invariant mass to peak at $105 \mathrm{GeV}$ or thereabouts. However, the peak is much smeared out because of the jet-merging effects. There is a further smearing due to addition of the contribution from events with a lepton and a neutrino - in these the neutrino carries away some energy, shifting the invariant mass peak lower than expected from the chargino mass alone. Further smearing effects could come from energy rescaling and detector effects, though these are not done here. Our final result is illustrated in Figure 7, where the solid line represents the four-jet events predicted in each bin of width $3.15 \mathrm{GeV}$. The parameters are chosen to match the first column of Table 1 . (We have checked that the distribution does not change much for the other two columns.) The distribution showed by the solid line includes the SM distribution which is also shown separately by the dashed line. The dotted lines show the actual data observed by ALEPH (Figure 2(a) of Ref. [2] ). It is clear that while our distribution is an improvement on the SM, it is too broad to be a viable explanation of the observed events. In fact, the distribution shown has about 21 events, since the reduction of $60 \%$ is not included. With this cut, the distribution would look even flatter. The probablity that the observed distribution is a fluctuation from our prediction is of the order of $10^{-3}$, which is somewhat better than the SM case, but not large enough for this possibility to be taken seriously as an explanation of the ALEPH four-jet events. Accordingly we conclude that the pair-production of charginos and their decays as conceived here cannot explain the ALEPH four-jet anomaly. This conclusion is not as robust as that for neutralinos, because the distributions may change when cuts are applied on charged track multiplicity and the sum of jet invariant masses, but it is unlikely that these will change the flat distribution so radically as to afford an explanation of the sharply-peaked data.

Finally we consider the possibility of seeing hard leptons $(e, \mu)$ and missing energy in conjunction with multijets which could be an additional test of the chargino signal in the current data sample. We have checked that barely a single event is predicted for the dilepton + jets + missing energy signal. Figure 8 shows the distribution of the single lepton + jets signal in different multijet channels with a cut of $10 \mathrm{GeV}$ on the minimum energy of the lepton. The largest number is predicted in the three-jet channel. This varies from 2.8 to 1.8 as the isolation criterion is changed from $\Delta R=0.4$ to 0.6 . This channel has a larger QCD 
background than the four-jets channel, which, however, has a smaller number of events. As the isolated lepton signal is easier to detect, one may expect it to be seen in all the detectors, so that the actual numbers should be multiplied by a factor of about 4 . It may be interesting to see if these signals can be isolated from the SM background, which would arise principally from $b \bar{b}$ production since, in most of the cases, the lepton lies just outside the nearest jet.

In Figure 9 we exhibit the isolation of the single hard lepton from the nearest jet for each multijet channel. As we have just noted, it is interesting that most of the leptons lie within this nearest jet or very close to it. Since most of the jets in this analysis arise from merging, one should expect the jets to be rather fat and hence the choice of the cutoff value of $\Delta R_{\min }$ may be taken as 0.5 or even 0.6 rather than the canonical choice of 0.4 . In that case it may be even more difficult to isolate the chargino signal from the SM background. Any further analysis of this, however, would require a more detailed simulation of jets and is outside the scope of the present work.

\section{SUMMARY AND CONCLUSIONS.}

To summarise, then, we have considered two possible explanations of the observed excess in four-jet events in $e^{+} e^{-}$collisions at LEP-1.5. The pair-production of (lightest) neutralinos, followed by their decays to three jets apiece through baryon number-violating $\lambda^{\prime \prime}$ couplings and subsequent merging of these jets to yield a four-jet signal compatible with the observed one turns out to be a non-starter since practically all the events are lost in the kinematic cuts. On the other hand, the more complicated case of (lighter) chargino pair-production and their decay to (lightest) neutralinos and jets and/or non-isolated leptons, followed by baryon number violating decays of these neutralinos, with jets merging as before, yields numbers adequate to explain the observed excess. This is essentially because the raw cross-section for chargino pair-production in $e^{+} e^{-}$collisions is much larger than the corresponding crosssection for neutralinos; though a large number of events are indeed lost through the kinematic cuts, we are left with interesting numbers in the final analysis. However the event shapes turn out to be too different from the observation for this option to be a good explanation of the four-jet anomaly.

Before concluding, we would like to point out two caveats to the results presented in 
this paper. One is the obvious requirement that more data need to be analysed so that the observed excess is put on firm ground and we have a clearer idea about the event shape. This is more so because the other experiments at LEP have not observed [1] any such excess. Unfortunately LEP has already gone on to higher energy runs, so such data analyses do not seem to be forthcoming. The other caveat is the more technical point that this analysis requires to be repeated (for the parameter space of interest which is mapped in Figure 4) with a more detailed simulation of the jet kinematics and detection efficiencies since the parton-level algorithm for jet-merging is at best representative.

In spite of the relative crudity of our analysis, however, we have been able to establish that the simplest application of $R$-parity violation to explain the four-jet anomaly is inadequate. Though indeed chargino pair-production can yield sufficiently large cross-sections to give the four-jet excess, it leads to broad distributions in the four-jet invariant mass which cannot explain the sharply peaked distribution discovered by the ALEPH Collaboration. A refined analysis using jet fragmentation and detector simulations is not likely to change the qualitative result, though the actual numbers may change. One therefore has to look for some other explanation of the anomaly than the one studied here. In fact, it seems from our analysis that any explanation which involves jet-merging will lead to smeared distributions and the best bet seems to be to consider two-jet decays of each of the produced particles, whatever they may be [21. We therefore conclude on a negative note. Though several interesting features have come up during the analysis, at least one candidate solution to the four-jet problem seems to be unacceptable. 


\section{Acknowledgements.}

The authors are grateful to the organisers of the Fourth Workshop on High Energy Particle Phenomenology, Calcutta where the idea for this work originated. They would also like to thank M. Bisset, D. Choudhury, M. Drees and X. Tata for discussions and some important suggestions. DKG acknowledges financial support from the University Grants Commission, Government of India. The work of RMG is partially supported by a grant (No: 3(745)/94/EMR(II)) of the Council of Scientific and Industrial Research, Government of India, while that of SR is partially funded by a project (DO No: SERC/SY/P-08/92) of the Department of Science and Technology, Government of India. 


\section{References}

[1] D. Buskulic et al (ALEPH Collaboration), Phys. Lett. B373 (1996) 246 and CERN preprint and CERN-PPE/96-45 (1996); P. Abreu et al (DELPHI Collaboration), CERN preprint CERN-PPE/96-003; M. Acciarri et al (L3 Collaboration), CERN preprints CERN-PPE/95-191 and CERN-PPE/96-29; G. Alexander et al (OPAL Collaboration), CERN preprints CERN-PPE/96-020 and CERN-PPE/96-025.

[2] D. Buskulic et al (ALEPH Collaboration), CERN preprint CERN-PPE/96-052 (1996), to be published in Z. Phys. C.

[3] S. Weinberg, Phys. Rev. D26 (1982) 287; N. Sakai and T. Yanagida, Nucl. Phys. B197 (1982) 533.

[4] J.L.Goity and M. Sher, Phys. Lett. B346 (1995) 69; other bounds can be found in C. S. Aulakh and R. N. Mohapatra, Phys. Lett. B119 (1982) 316; L. J. Hall and M. Suzuki, Nucl. Phys. B231 (1984) 419; S. Dawson, Nucl. Phys. B261 (1985) 297; S. Dimopoulos and L. J. Hall, Phys. Lett. B207 (1987) 210; V. Barger, G. F. Giudice and T. Han, Phys. Rev. D40 (1989) 2987; L. J. Hall, Mod. Phys. Lett. A5 (1990) 467; S. Dimopoulos et al, Phys. Rev. D41 (1990) 2099; D. P. Roy in Proceedings of the Tenth DAE Symposium on High Energy Physics, Bombay (1992); G.Bhattacharyya and D. Choudhury, Mod. Phys. Lett. A10 (1995) 1699; G.Bhattacharyya, J. Ellis and K. Sridhar, Mod. Phys. Lett. A10 (1995) 1583; G.Bhattacharyya, D. Choudhury and K. Sridhar, Phys. Lett. B355 (1995) 193; D. Choudhury and P. Roy, TIFR preprint no. TIFR/TH/96-12, hep-ph/9603363 (1996); R. M. Godbole, P. Roy, X. Tata, Nucl. Phys. B401 (1993) 67; P. D. Acton et al (OPAL Collaboration), Phys. Lett. B313 (1993) 333; V. Barger, W. Y. Keung, and R. J. N. Phillips, Phys. Lett. B356 (1995) 546; D. Buskulic et al (ALEPH Collaboration), Phys. Lett. B349 (1995) 238; J. C. Romão et al, Univ. of Valencia preprint no. FTUV/96-06, hep-ph/9604244 (1996); D.K.Ghosh, S. Raychaudhuri and K. Sridhar, hep-ph/9608352.

[5] H. Dreiner and P. Morawitz, Nucl. Phys. B428 (1994) 31. 
[6] H. Dreiner, S. Lola and P. Morawitz, hep-ph/9606364 (1996).

[7] See, for example, A. Yu. Smirnov and F. Vissani, ICTP preprint IC-96-16, hepph/9601387 (1996), and references therein.

[8] The stategy of considering a single $R$-parity violating coupling at a time was first suggested by Barger, Giudice and Han (see [5]). The choice of $\lambda_{212}^{\prime \prime}$ is suggested in H. Baer, C. Kao and X. Tata, Phys. Rev. D51, 2180 (1995). For a recent re-evaluation, see H. Baer, C.-H. Chen and X. Tata, hep-ph/9608221.

[9] D. Buskulic et al (ALEPH Collaboration) in Ref. [5]; G. Alexander et al (OPAL Collaboration) Phys. Lett. B313 (1993) 333.

[10] V. Barger et al in Ref. [5].

[11] S. Banerjee et al, work in progress.

[12] P. Antilogus et al (LEP Electroweak Working Group), CERN preprint CERN-PPE/95$172(1995)$.

[13] H. Baer, M. Drees and X. Tata, Phys. Rev. D41, 3414 (1991).

[14] G. Arnison et al (UA1 Collaboration), Phys. Lett. B123 (1983) 115, ibid. 132 (1983) 214; C. Albajar et al (UA1 Collaboration), Nucl. Phys. B309 (1988) 405; N. J. Hadley (D0 Collaboration) D0 note 904 (Nov. 1989); H. J. Daum et al, Z. Phys. C8 (1991) 167; S. S. Snyder (D0 Collaboration), Ph. D. thesis (1995).

[15] D. A. Dicus et al, Phys. Rev. Lett.51 (1983) 1030; S. Dawson, E. Eichten and C. Quigg, Phys. Rev. D31 (1985) 1581; A. Bartl, H. Fraas and W. Majerotto, Nucl. Phys. B278 (1986) 1; X. Tata and D. A. Dicus, Phys. Rev. D35 (1987) 2110; R. Barbieri et al, Phys. Lett. B195 (1987) 500; M. Chen et al, Phys. Reports159 (1988) 201; H. Baer et al, Int. J. Mod. Phys. A4 (1989) 4111. S. Ambrosanio and B. Mele, Phys. Rev. D53, 2541 (1996); ibid. 52,3900 (1995).

[16] D. P. Roy, Phys. Lett. B283, 270 (1992). 
[17] H. Dreiner, M. Guchait and D. P. Roy, Phys. Rev. D49, 3270 (1994).

[18] F. Abe et al (CDF Collaboration), Phys. Rev. Lett.76 (1996) 2006; S. Abachi et al (D0 Collaboration) Phys. Rev. Lett.75, 618 (1995).

[19] H. Baer et al, in Ref. [15]; A. Bartl, H. Fraas and W. Majerotto, Z. Phys. C30 (1986) 441; M. Chen et al, in Ref. 15]; D. A. Dicus et al, in Ref. [15]; S. Dawson et al, in Ref. [15]; P. Nath, R. Arnowitt, and A. Chamseddine, Harvard Report HUTP -83/A077 (1983); V. Barger et al, Phys. Lett. B131 (1983) 372; J. Ellis et al, Phys. Lett. B127 (1983) 233, ibid., B132 (1983) 463.

[20] See Bartl et al, in Ref. [19].

[21] P. H. Chankowski, D. Choudhury and S. Pokorski, hep-ph/9606415; D. Choudhury and D.P. Roy, CERN preprint CERN-TH-96-203, hep-ph/9608264. 


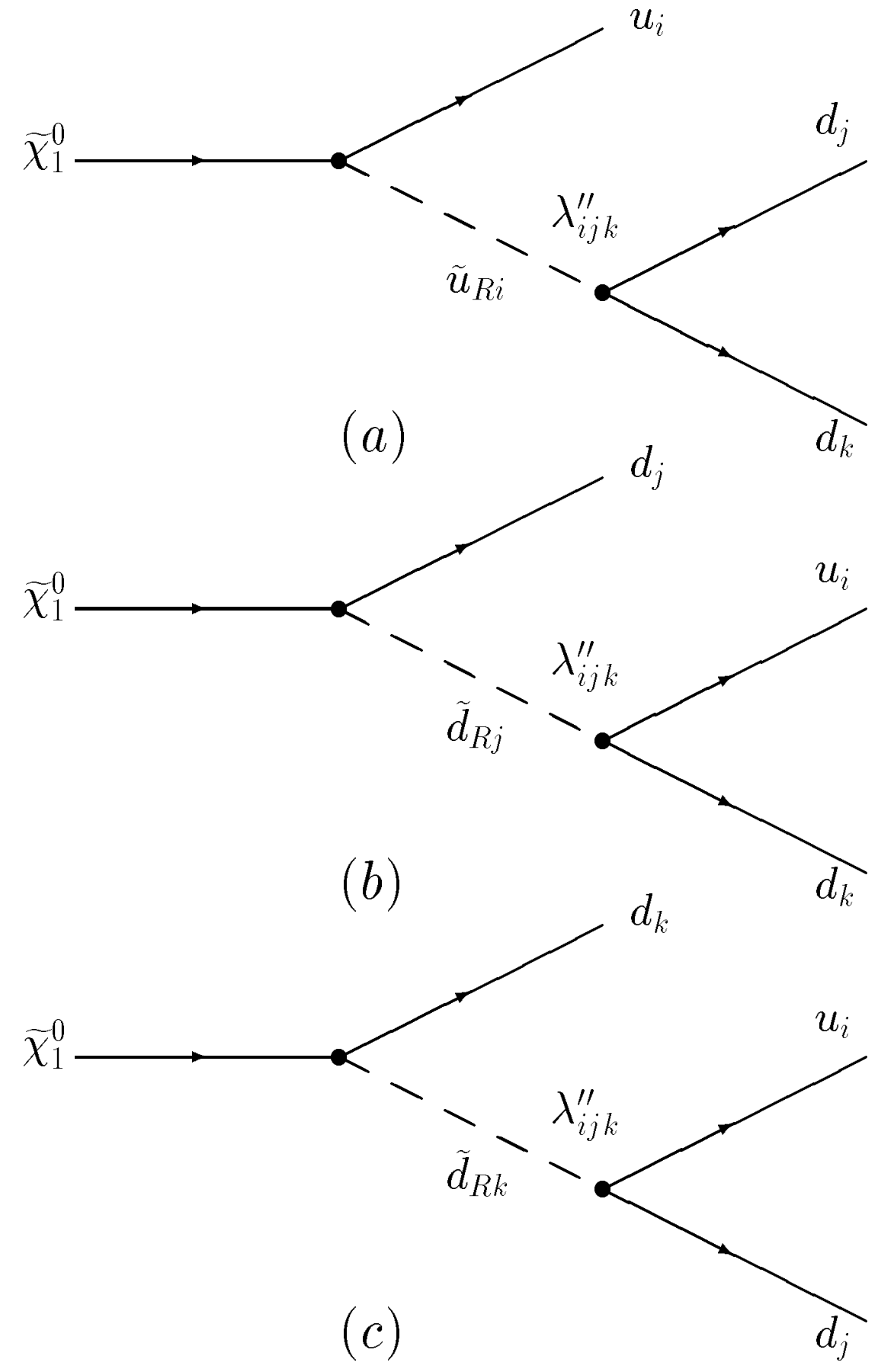

Figure 1. Feynman diagrams contributing to the decay of the LSP $\left(\widetilde{\chi}_{1}^{0}\right)$ for a baryon numberviolating $\lambda_{i j k}^{\prime \prime}$ coupling. Only right squarks contribute to this process. 


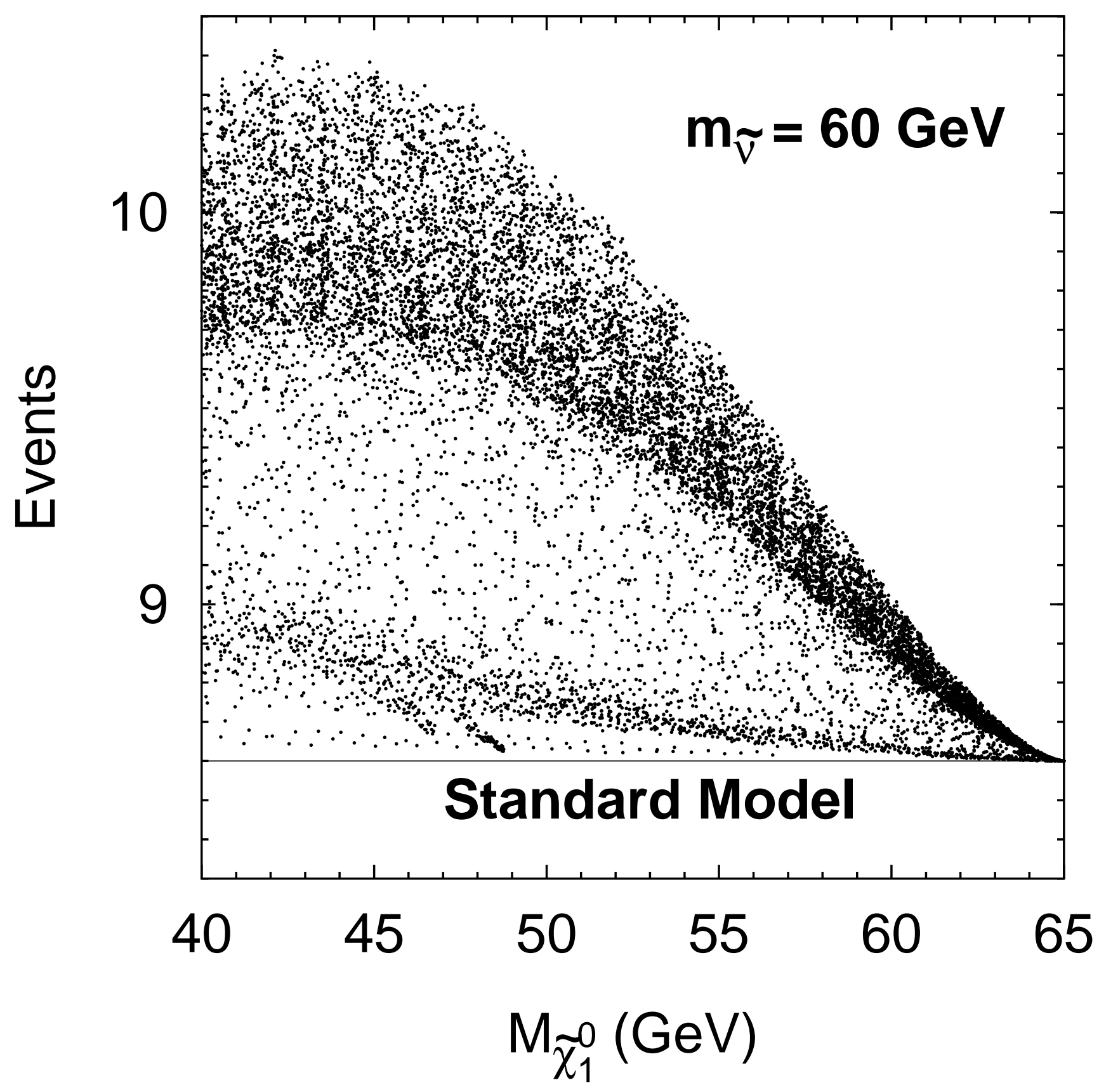

Figure 2. Scatter plot showing the number of four-jet events arising from (lightest) neutralino pair-production against the mass of the neutralino for values of $M_{\widetilde{g}}, \mu, \tan \beta$ allowed by LEP-1 constraints (assuming $5.7 \mathrm{pb}^{-1}$ luminosity). We set the sneutrino and right selectron masses to $m_{\widetilde{\nu}}=m_{\widetilde{e}_{R}}=60 \mathrm{GeV}$ and the squark mass $m_{\widetilde{q}}=300 \mathrm{GeV}$. The cross-section decreases as the former increases and is insensitive to the latter. 


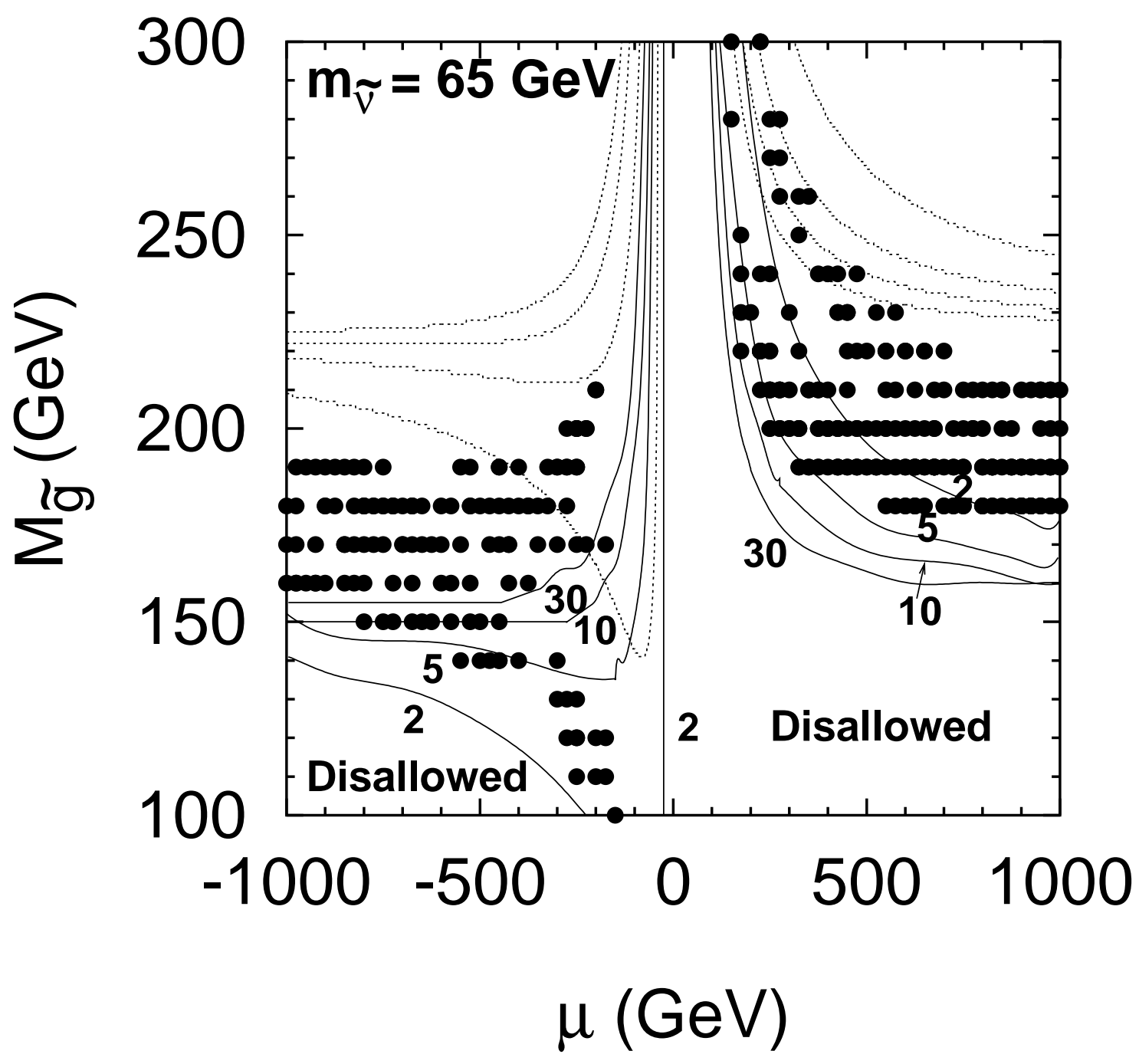

Figure 3. Bullets mark points corresponding to $(16 \pm 0.5)$ SM plus chargino-induced four-jet events and $50 \mathrm{GeV}<M_{\tilde{\chi}_{1}^{+}}<55 \mathrm{GeV}$. Each point is obtained with some value of $\tan \beta$ among the set $\tan \beta=1.5,2,5,10,15,20,25,30,35 . M_{\widetilde{g}}(\mu)$ is sampled in steps of 10 (25) GeV. Solid lines bound the LEP-1 excluded regions for marked values of $\tan \beta$ and the dotted lines bound the region kinematically accessible to LEP-1.5 for the same values of $\tan \beta$. Dotted and solid curves rise (fall) with $\tan \beta$ in the left (right) half-plane. 


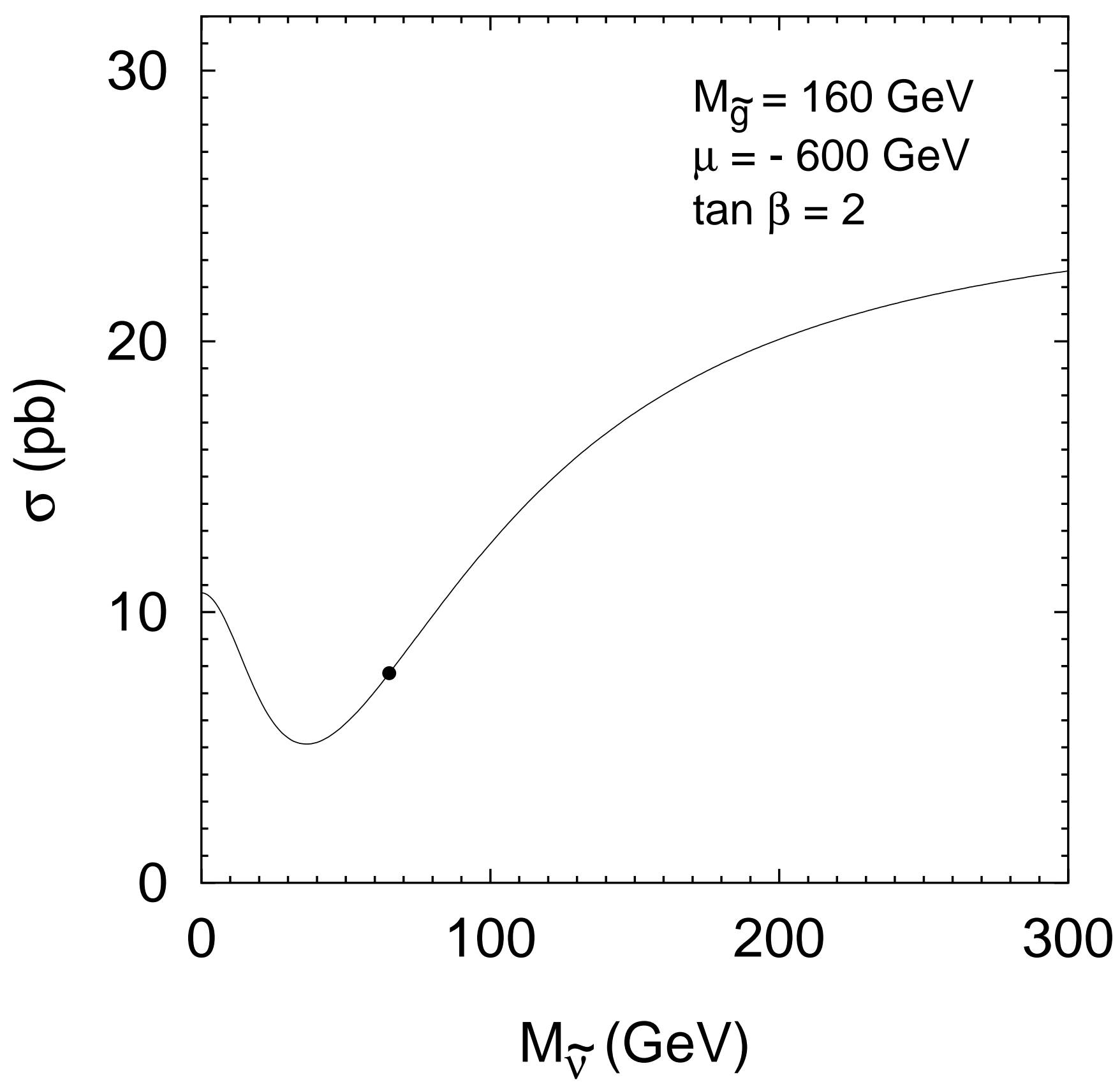

Figure 4. Cross-section for pair-production of the lightest chargino as a function of sneutrino mass. The parameters are chosen to agree with the first column of Table 2 . The bullet shows the value of sneutrino mass which yields the numbers in Figures 3 and 5 and in Table 2. 


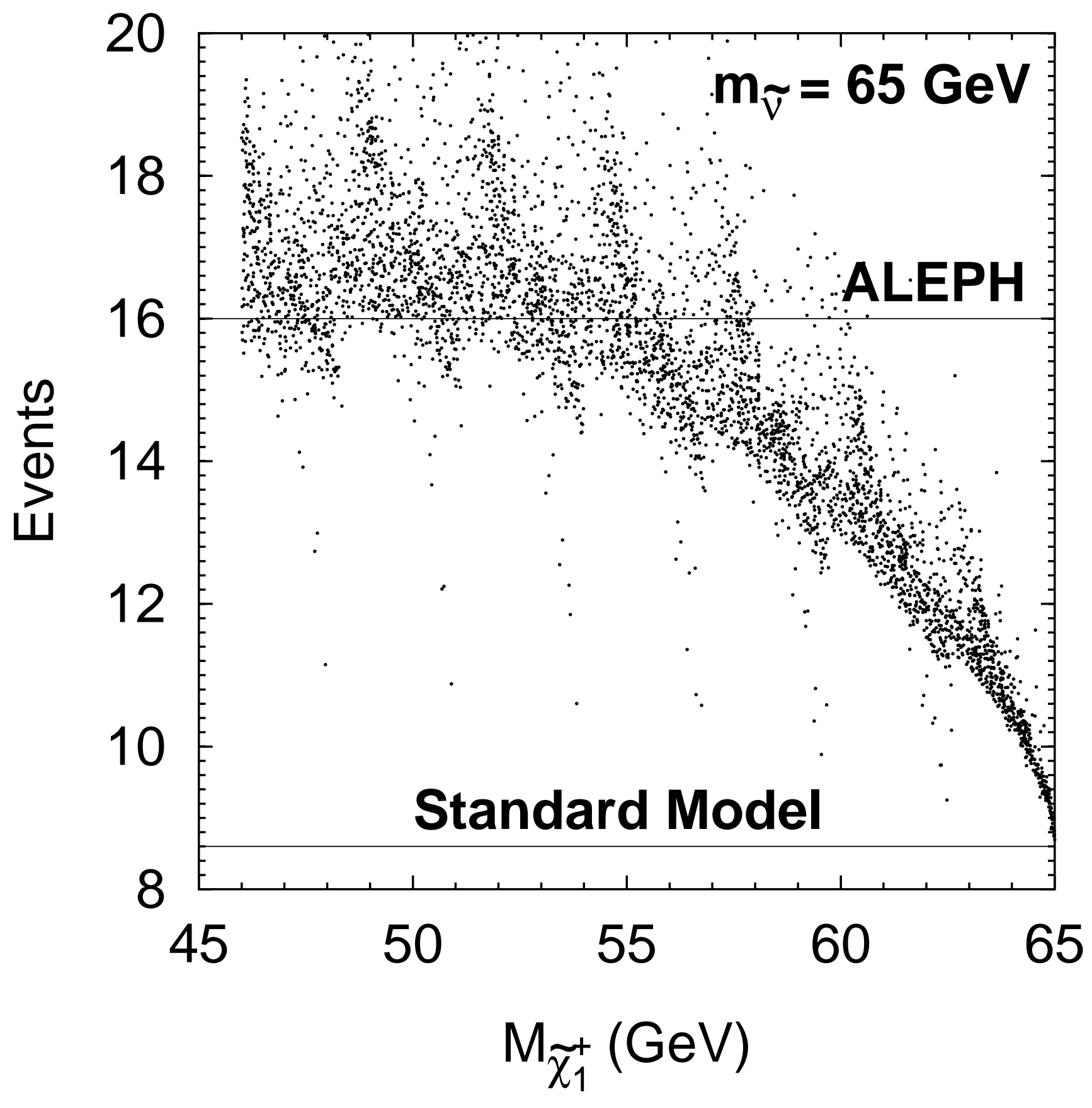

Figure 5. Scatter plot showing the number of four-jet events arising from pair-production of the lighter chargino against its mass for values of $M_{\widetilde{g}}, \mu, \tan \beta$ allowed by LEP-1 constraints. We set the sneutrino mass to $65 \mathrm{GeV}$ and the squark mass to $500 \mathrm{GeV}$. The cross-section increases with $m_{\widetilde{\nu}}$ in the allowed region and is insensitive to $m_{\tilde{q}}$. 


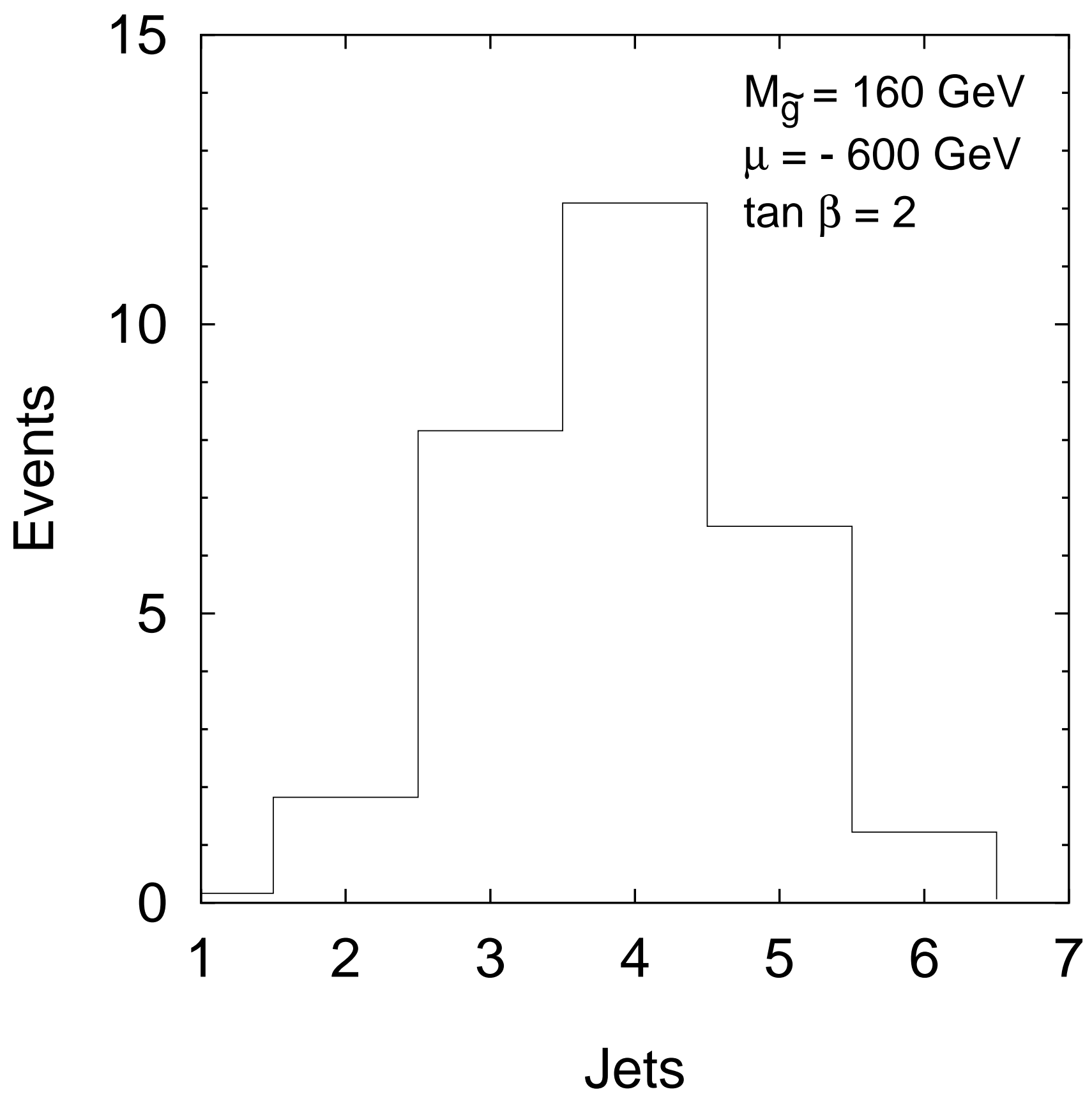

Figure 6. Distribution of events in multijet channels for production of chargino pairs corresponding to the first column of Table 2 and $5.7 \mathrm{pb}^{-1}$ luminosity. Cuts on $M_{i j}$ and $\Delta M$ are not imposed. The $40 \%$ reduction assumed for charged track multiplicity and jet invariant mass cuts is also not applied. 


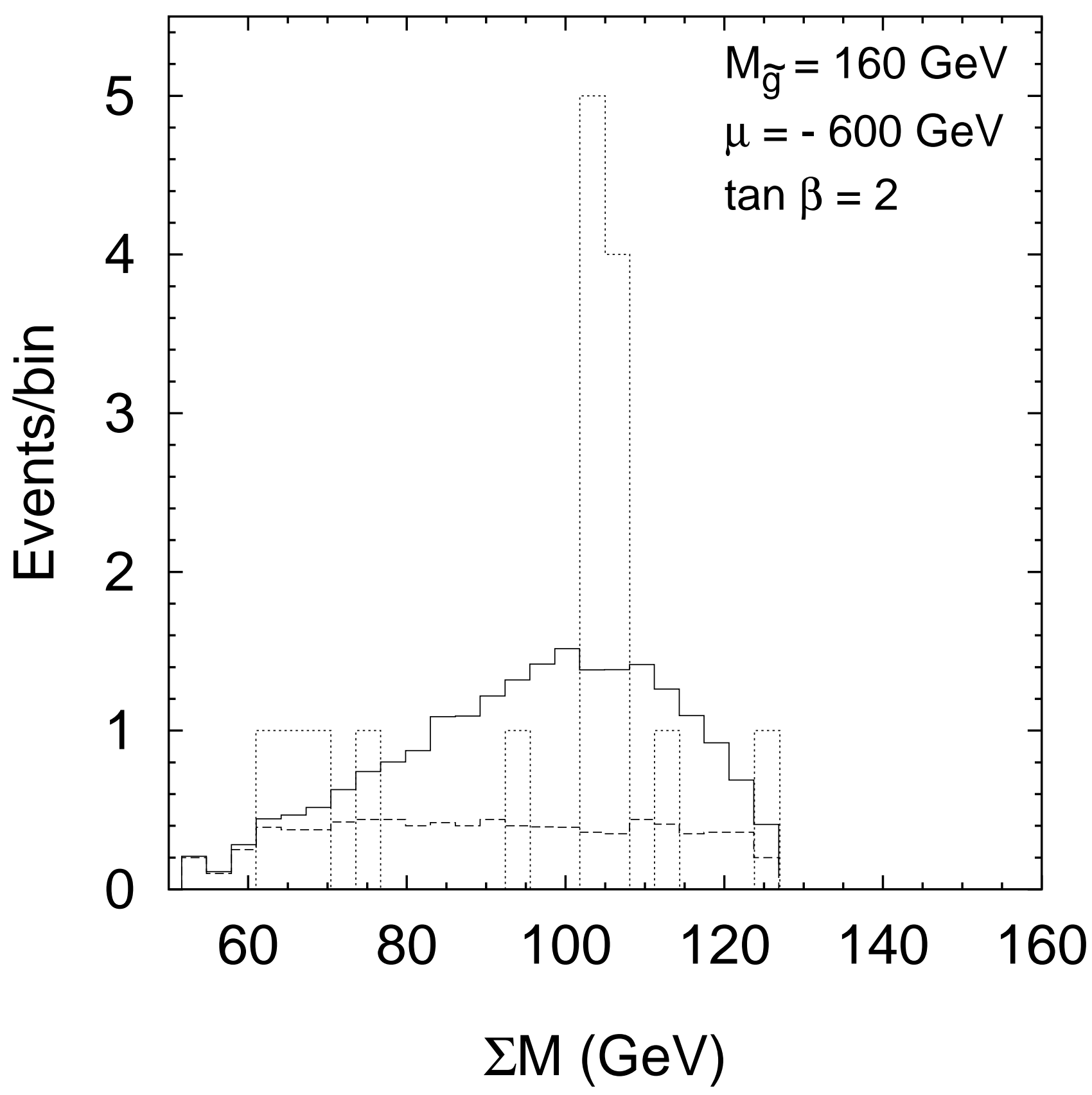

Figure 7. Distribution of four-jet events against the four-jet invariant mass $\Sigma M$ for $5.7 \mathrm{pb}^{-1}$ luminosity. The solid line represents the prediction from chargino pair-production for the parameters in the first column of Table 2. The dotted line represents the actual observation of the ALEPH group while the dashed line represents the SM background (after Fig. 2 of Ref. [2]). The $40 \%$ reduction assumed for charged track multiplicity and jet invariant mass cuts is not applied. 


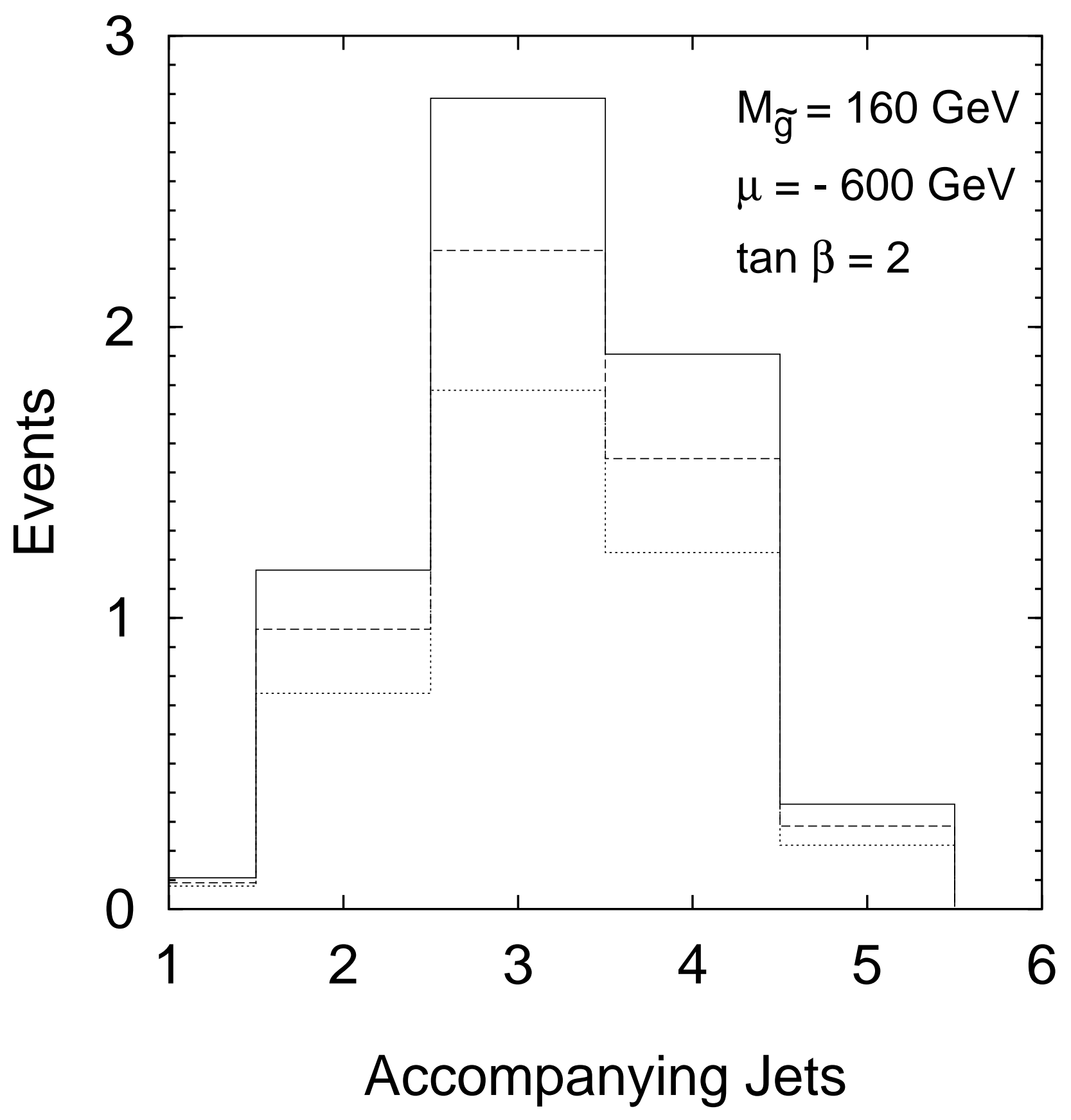

Figure 8. Distribution of jets accompanying a single isolated lepton for $5.7 \mathrm{pb}^{-1}$ luminosity for the parameters in the first column of Table 2. Solid, dashed and dotted lines are obtained with isolation cuts of $\Delta R>0.4,0.5$ and 0.6 respectively between the lepton and the nearest jet. There is a cut of $10 \mathrm{GeV}$ on the minimum energy of the lepton. 


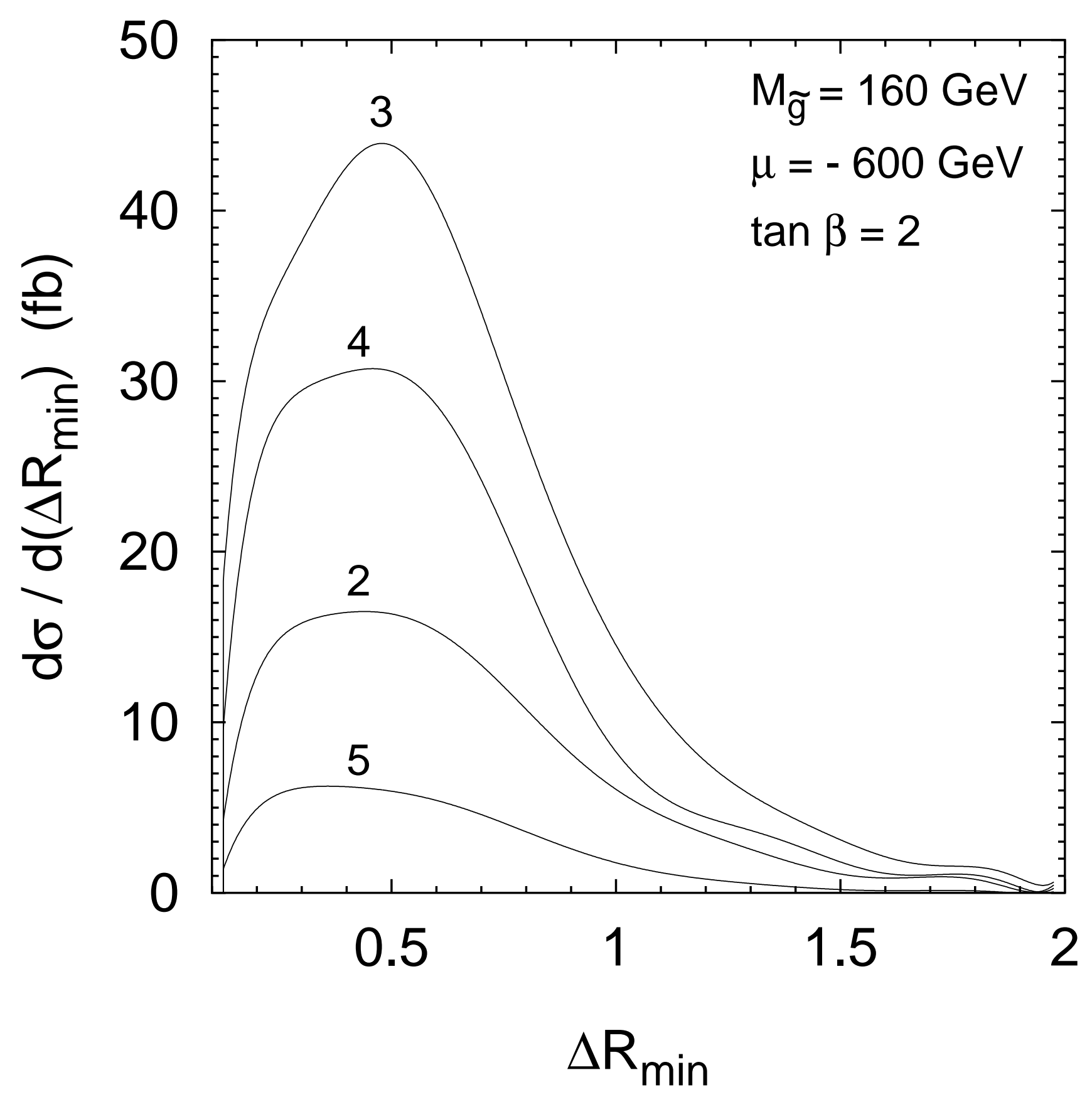

Figure 9. Illustrating the angular separation between a single lepton and the nearest jet for 2,3,4,5 (marked next to the appropriate curve) accompanying jets. Parameters are chosen as in Figure 8. There is a cut of $10 \mathrm{GeV}$ on the minimum energy of the lepton. 